\title{
Commercial offer for cardiac centers and potential investors
}

\author{
Vladimir I. Ermoshkin ${ }^{1 *}$ \\ ${ }^{1}$ Research and Development Center “RESONANS” Ltd, Russia \\ Dear medical specialists! \\ Dear potential investors! \\ Dear patients!
}

You are, of course, aware about the actual situation in cardiology and in some other related fields of medicine. For a long time the mortality from cardiovascular (CVD) and oncological diseases are in the first places in developed countries.

We can state: although there are some relative successes, but in general, the theoretical and practical cardiology are all together in stagnation, in crisis [1]. Most people die prematurely because of CVD, and in recent decades, the probability of sudden cardiac death in young people of 30-60 years, has increased.

I believe that we have a critical situation for now, and any new idea, any new hypothesis on the mechanism of CVD should be treated more carefully, because the modern theoretical cardiology has not been developed for decades. I ask you to pay a very close attention to my New Theory of CVD. Let me inform you that several well-known cardiologists know about my New Theory, none of them have yet been able to refute it, but no one has agreed to put some experiments for evidence.

The World Health Organisation speaks about the epidemic of CVD, so do the reputable scientists [2], I hear about it at medical conferences [3,4]. For a long time, there are no breakthroughs in cardiology. Perhaps, therefore, for the entire time of the existence of the Nobel Prize, i.e., for 117 years, there is not a single award for cardiology. The award for the invention of ECG in 1924 and the award for the discovery of the role of nitric oxide as a signal molecule in the regulation of the cardiovascular system were received in 1998 by an optometrist and a pharmacist from Herbalife. The names of the lucky ones: Willem Einthoven and Luis Ignarro. For comparison: during the same time, the researchers of infectious diseases received "Nobel" 48 times! Are these results accidental? I don't think so. And that's why.

In fact, the epidemic of CVD is widely spreaded in the world and, interestingly, the causes of almost all CVD are officially considered unknown, or rather, most diseases have a "multi factor" pathogenesis. Studying the primary sources, one can note that the causes and attendant factors for each heart disease are repeated from one cardiovascular disease to another, the treatment and prevention of some CVD are also largely identical.

Well, if this is so, then the mechanism of CVD development is the same, but each CVD has its own specifics, after all, each person is also with his "specifics"? The mechanism is simply hidden from the eyes of doctors and researchers because of previous erroneous assumptions,

\begin{abstract}
"evidences and discoveries."
Against this background, as a physicist, I, for 6 years, have been promoting my vision of the main mechanism of arrhythmia, heart failure, causes of venous plethora, metabolic syndrome, other diseases and acquired pathologies. I believe that the official cardiology over the past 50-100 years has committed several blatant mistakes that do not allow us to get out of the medical impasse.
\end{abstract}

Until errors in cardiology are corrected, it is not realistic to hope for success in the treatment of heart disease.

I will give only two errors.

The first error. When considering the causes and mechanisms of arrhythmia, the possibility of exciting cardiomyocytes not only with the help of bioelectric excitations from the sinus node, but also with the help of mechanical waves propagating through the vessels and the myocardium, have been forgotten for decades. Developing my theory, I realized that the most dangerous kind of arrhythmia "attack of ventricular fibrillation" is nothing like, usually under conditions of ischemia, simultaneous launch of several excitations of different nature and position of sources. At the same time, the electrical excitations produce mechanical waves and excitations, and mechanical waves create electrical excitations, so the chaos (random superposition) of myocardial excitations occurs throughout its scope. This phenomenon should be considered taking into account the rapid change of tissue refractory zones continuously in time. The mechanical waves propagate through refractory tissues and tissues ready for excitation, and the bio electric ones cannot propagate through refractory tissue, so they suddenly go out and reappear elsewhere (to the surprise of doctors) already due to pervasive mechanical excitations. Death occurs in 1-2 minutes due to a decrease in pumping function and critical ischemia of the main organs of man.

The second error. In most cases, an increase in venous pressure at the local level and edema occurs not because of certain "obstacles" in the way of movement of venous blood to the vena cava and to the heart, i.e., not because of thrombi and tumors, but because of open large arteriovenous anastomoses (LAA). The arterial blood passed through the anastomoses raises pressure not only in the hollow vein, but also over time, after damage to the venous valves, in some small veins up to the venules. As a result, there is a lack of a pressure gradient between arterioles and venules, which leads to a slowing of the capillary

Correspondence to: Vladimir I. Ermoshkin, Research and Development Center "RESONANS" Ltd, Russia; E-mail: evlad48@list.ru

Received: April 03, 2017; Accepted: April 12, 2017; Published: April 18, 2017 
circulation and to stasis. Over time, the venous fluid begins to penetrate through the walls of the vessels into the intercellular space where an edema occurs. But the medicine, explaining the stagnation of blood, swapped the consequence and the cause. The official medicine believes: first come clots and tumors, and then stagnation of venous blood.

Now, especially since May 2016, after the publication of two articles of mine in English in the journal Cardiocode, I receive letters (several E-mails every day) from popular (and not so) medical journals with the offer of publishing at least some of my new articles or comment to them. Many letters come with proposals to speak at conferences. Invitations have already been received from the USA, England, Ireland, Australia, the United Arab Emirates, Japan, Singapore, China, Canada, Italy, India, Spain, etc. But I do not even answer most of the letters, because I do not have any grant or sponsorship. My own funds are enough only to write and publish some articles.

Nevertheless, the last time I made a report in London at the EuroHeartFailure-2017 conference (March 15-17) [5,6].

I can say that after my reports there have been questions from many participants. But always the discussions end in a council: we need to conduct several experiments and show / prove that the New Theory is correct. But to hold means to overturn the old theory. Which of the leaders will take such a step? How to persuade the main specialists to make pressure on the cardiac authorities? After all, we need a laboratory together with specialists who know the technique, we need a clinical base. Financing is necessary, not for me, but at least for the experts who will conduct experiments. [7-16].

Details about my New Theory can be found by reading my articles

Now briefly about what needs to be confirmed or demonstrated so that the New Theory could be considered proven.

1) It is necessary to confirm that in a significant percentage of cases, the attacks of cardiac arrhythmia occur due to open large arteriovenous anastomoses (LAA). These phenomena lead to a reduction in blood pressure, overflow of the venous pool, increase in venous pressure in the vena cava, including in the right atrium. In some cases, the mechanically induced extra systoles or attacks of atrial or ventricular tachycardia can begin due to the run of mechanical impulses along the vena cava and further along the myocardium. In other words, under some conditions the mechanical pulse waves run with equal time intervals along a closed contour of vessels and support themselves cyclically due to mechanical excitations of the heart. It's here the answer to the surprising constancy of the RR interval on the ECG with paroxysmal atrial or ventricular tachycardia.

2) It must be confirmed that along with pressure increasing in the hollow veins in case of emergency leakage of arterial blood from the arteries to the veins with open large arteriovenous anastomoses (LAA), there is an increase in pressure in some medium and small veins, up to the venules. Such phenomena lead to blood stasis, damage to the valves, edema and thrombosis. The most vulnerable places for stagnation in upright person are the ankles of the legs and the area of the small pelvis. Due to hypodynamia and sedentary lifestyle, an entire range of diseases can arise, such as metabolic syndrome, heart failure, endometriosis in women, prostatitis in men, hemorrhoids, abdominal-visceral obesity. It is in the pelvic area, apparently not by chance, an increased likelihood of some types of cancer.

3) Apparently, it is necessary to develop devices to prevent mechanically induced arrhythmia attacks, for example, to develop suppressors of mechanical impulses in some vessels. It is also necessary to create smart "adjustable devices" such as artificial arteriovenous shunts to reduce peak blood pressure values in some organs to prevent strokes and heart attacks and venous plethora. Perhaps, then the need will decrease, especially for young people, in cauterization of "ectopic focus" by radiofrequency ablation, in putting stents, in coronary artery shunting, and in other types of surgeries. Perhaps, due to preventive measures, these operations will not be needed, or they will be transferred to a later period of life.

\section{Conclusions}

I can state that a significant interest in my ideas and suggestions in medicine is growing every month.

I think that the results of the forthcoming experiments will force to review many medical dogmas, to re-evaluate the effectiveness of "modern developments" in the prevention and treatment of most CVD and cancer.

It is necessary to develop new devices for preventing CVD and getting rid of sudden cardiac death with an absolutely healthy heart. Some devices already have sketches.

Man should live longer! But the old theoretical errors in medicine, admitted more than 50-100 years ago, do not allow this.

Why cut, cauterize or saturate the myocardium with chemistry, if doctors don't know the cause and act on a false theory.

I think my theory can be said this way: "If the large arteriovenous anastomoses (LAA) were not known at all, then they would have to be invented to explain the most of clinical observations and diseases that occur in humans!"

I urge investors to join in the search for new solutions. After all, the basis of the New Theory is already there, and if you take it seriously, then in 1-10 years you can achieve significant success!

I think that to continue my work, I need an entrepreneur, a businessman (maybe not one), familiar with medicine and ready to work with me, having loaded most of the burden. Medicine is in dire need of innovations, but it will be extremely difficult to break through the silent conspiracy of medical bosses. Sorry, but that's how I see this problem, that's it.

\section{References}

1. World Health Report (2002) Reducing risks, promoting healthy life. Geneva: World Health Organization.

2. Итоги. Пора лечить. N50/60 (10.02.07)

3. Голиков А.П. Актуальные проблемы кардиологии. 11.01.2016 http://heal-cardio ru/2016/01/11/aktualnye-problemy-kardiologii/

4. British Heart Foundation. Heart failure. Internet resource. 2017.

5. Ermoshkin VI (2017) The new theory of heart failure. London.

6. Ermoshkin VI. (2016) New theory of arrhythmia. Conceptual substantiation of arrhythmia mechanisms. Cardiometry 10: 6-17.

7. Ermoshkin VI. (2016) The mechanism of bronchial asthma. Why do the most serious asthma attacks occur at night? EC Cardiology 2: 196-199.

8. Ermoshkin VI. (2016) Arteriovenous anastomoses and cardiovascular diseases. $8^{\text {th }}$ Cardiovascular Nursing \& Nurse Practitioners Meeting. August 08-09, 2016 Las Vegas, USA.

9. Ermoshkin VI. (2016) A New Theory of Certain Cardiovascular Diseases. EC Cardiology 2.

10. Ermoshkin VI. (2017) Venous congestion due to large arteriovenous anastomoses. Hypertens Curr Concepts Ther 1. 
11. Ermoshkin V. (2017) The Cause of Some Cancers because of the Open Arteriovenous Anastomoses. J Gastrointest Cancer Stromal Tumor 2: 1000111.

12. Ermoshkin VI Problems of heart failure. Unexpected outcome. London, 15-17.03.2017.

13. Ermoshkin VI. Arrhythmia and Cardiac Surgery. July 14-16, 2016, Brisbane, Australia.

14. Ермошкин В.И. НОВАЯ ГИПОТЕЗА АРИТМИИ СЕРДЦА У ЧЕЛОВЕКА. Стр 73. «Кардиостим-2016», Санкт-Петербург, 18 - 20 февраля 2016
15. Ermoshkin VI. (2017) The new theory of cancer complements ancient Chinese Qigong therapy. Hypertens Curr Concepts Ther 1 .

16. Ermoshkin VI. (2016) Heart transplantation mysteriously eliminates arrhythmia Cardiometry 18-21.

Copyright: (C2017 Ermoshkin VI. This is an open-access article distributed under the terms of the Creative Commons Attribution License, which permits unrestricted use, distribution, and reproduction in any medium, provided the original author and source are credited. 